\title{
Estudio observacional de la distensión de la grasa plantar en pacientes sanos en sedestación, carga bipodal y monopodal
}

\author{
Observational study of the plantar fat pad distension in healthy patients in sitting position, \\ bipodal and monopodal standing
}

Javier Ramos-Ortega' ${ }^{1}$ Juan Agustín Martín Jiménez², María José Manfredi Márquez³, Gabriel Domínguez Maldonado ${ }^{1}$ y Bárbara Pineda Bascón ${ }^{4}$

'Departamento de Podología. Universidad de Sevilla. Sevilla, España. ${ }^{2}$ Centro de podología. Mairena del Alcohor, Sevilla, España. ${ }^{3}$ Centro de Podología. Pilas, Sevilla, España. ${ }^{4}$ Clínica del Pie Bami, Sevilla, España

Palabras clave:

Grasa plantary distensión, sedestación carga bipodal, carga monopodal, ángulo de Clarke, índice de Chippaus-Smirack, índice Staheli.

\section{Resumen}

Introducción: Este trabajo se planteó con el objetivo de estudiar la variación de la distensión de la grasa plantar en sedestación, carga bipodal y monopodal, y relacionar estos cambios con la huella plantar y la postura del pie.

Pacientes y métodos: Participaron en el estudio 14 adultos sanos a los que se les escaneó la huella plantar en carga bipodal, en carga monopodal y en sedestación. En estas huellas se midieron el ángulo de Clarke, índice de Chippaux-Smirack, índice de Staheli, anchura del antepié, anchura del itsmo y anchura del talón, y se compararon estas medidas entre las tres situaciones de carga.

Resultados: Para el ángulo de Clarke, el índice de Chippaux-Smirack y el índice de Staheli se observaron diferencias significativas en todas las mediciones, excepto la comparación entre sedestación y bipedestación bipodal en el índice de Staheli. Las correlaciones en la modalidad bipodal-monopodal fueron todas fuertes $(r>0.8)$. Para las anchuras del antepié, del istmo y del talón en las tres posiciones, obtuvimos diferencias estadísticamente significativas en todas las mediciones. Se obtuvo correlación fuerte directa entre FPI y ángulo de Clarke en carga monopodal ( $r=0.69)$, y correlación fuerte inversa entre FPI y ángulo de Staheli en carga bipodal.

Conclusiones: Los resultados del estudio sugieren que existen cambios en la huella plantar en las diferentes posiciones. Se observan cambios en la misma, a medida que aumenta la carga.

\section{Keywords:}

Plantar fat, distention sitting, standing, monopodal standing Clarke angle, ChippauxSmirack index, Staheli index.

\section{Abstract}

Introduction: This work aimed at studying the variation of the distention of plantar fat in sitting, bipodal and monopodal standing, and to relate these changes with the footprint and the posture of the foot.

Patients and methods: 14 healthy adults participated in the study. Their plantar footprint was scanned in bipodal standing monopodal standing, and seated. In these footprints, the Clarke angle, Chippaux-Smirack index, Staheli index, forefoot width, itsmo width, and heel width, were measured. And these measures were compared between the three weightbearing conditions.

Results: For the Clarke angle, the Chippaux-Smirack index and the Staheli index, significant differences were observed in all measurements, except the comparison between sitting and bipodal standing in the Staheli index. The correlations in the bipodal-monopodal modality were all strong $(r>0.8)$. For the forefoot, isthmus and heel widths in all three positions, we obtained statistically significant differences in all measurements. Direct strong correlation between FPI and Clarke angle in monopodal weightbearing ( $r=0.69$ ) was obtained, and strong inverse correlation between FPI and Staheli angle in bipodal weightbearing.

Conclusions: The study results suggest that there were changes in the plantar footprint in the different weightbearing conditions as the load increased. 


\section{INTRODUCCIÓN}

El talón es la parte del pie que primero contacta con el suelo y, por ello, la primera en recibir las fuerzas de reacción" Cuando este entra en contacto con el suelo, las fuerzas que soporta son aproximadamente del $85-110 \%$ del peso corporal, llegando a un 250 \% en la carrera ${ }^{2}$. En un varón de 70 kilogramos, el área del talón tiene un promedio de $23 \mathrm{~cm}^{2}$, y la presión que puede alcanzar es de $3,3 \mathrm{~kg} / \mathrm{cm}^{2}$. Esta presión puede alzar máximos en algunas áreas de $5 \mathrm{~kg} / \mathrm{cm}^{2}$ e incluso el doble durante la carrera ${ }^{3}$. Además, se calcula que durante la marcha se producen unos 721 choques de talón por kilómetro recorrido 4 .

La almohadilla grasa plantar (AGP) cubre desde el calcáneo ${ }^{5}$ hasta la zona metatarsal ${ }^{6}$, funcionando como un amortiguador $^{5}$. Juega un papel importante en el soporte del peso corporal y permite que la marcha se produzca sin dolor. Esto se debe principalmente a su estructura compuesta por glóbulos de grasa encapsulados en estructuras fibroelásticas reticuladas 6 . El deterioro de la almohadilla grasa plantar juega un papel importante en la patogénesis tanto de metatarsalgias ${ }^{7}$ como de los síndromes que causan dolor en el talón ${ }^{8}$.

Las metatarsalgias y los síndromes dolorosos del talón son patologías comunes que a diario se presentan en consulta, y que en muchas ocasiones su origen y diagnóstico resulta controvertido $^{5,9}$. En la actualidad existen estudios que hablan del desgaste o la pérdida de la almohadilla grasa plantar como causa de metatarsalgias o del síndrome doloroso del talón ${ }^{4,8-11}$. Los estudios se centran principalmente en la observación de los cambios producidos en el grosor y sus propiedades ${ }^{1,3,4,8-12}$.

En un trabajo realizado en Australia sobre la prevalencia y correlación del dolor de pies de una población en el que se escogieron al azar 3206 personas, un 3,6\% presentaba dolor en el talón ${ }^{13}$, y estudios americanos indican que hasta entre un 7 y un $10 \%$ de las personas adultas sufren dolor talar ${ }^{14}$.

Un grupo de población en el que debemos tener muy en cuenta el mantener las propiedades de la almohadilla grasa plantar en correctas condiciones es sobre los pacientes diabéticos. Las úlceras por presión en el talón son la segunda localización más frecuente se localizan este tipo de lesiones ${ }^{15}$.

El conocimiento de los cambios en la anchura de la grasa plantar a través de la huella sobre sujetos sanos puede ofrecernos datos de normalidad que después se utilizarían con fines preventivos sobre diferentes grupos de pacientes (diabéticos, pacientes con alteración neurológica, pacientes con dolor en el talón, etc.). Determinar estos cambios a través de la huella es un método sencillo y económico, aplicable en cualquier situación por medio de una pedigrafía.

Es por ello que nos planteamos una investigación para estudiar los posibles cambios que se presenten en la huella plantar sobre pacientes sanos, tanto en sedestación como en bipedestación monopodal y bipodal. Además, se utiliza la postura del pie para comprobar la correlación existente entre los diferentes tipos de pies y el comportamiento de la almohadilla grasa situada bajo la planta del pie.

Los objetivos de nuestro estudio fueron estudiar la variación de la distensión de la grasa plantar en sedestación, carga bipodal y monopodal; analizar la modificación del ángulo de Clarke, índice de Chippaux-Smirack, índice de Staheli en sedestación, carga bipodal y monopodal; y determinar la relación entre los cambios de la huella plantar y la postura del pie.

\section{PACIENTES Y MÉTODOS}

Es un estudio descriptivo, transversal y observacional, enmarcado dentro de un estudio piloto ${ }^{16}$.

La muestra global del estudio está compuesta por 14 personas ( 7 hombres y 7 mujeres) con edades comprendidas entre los $24.43 \pm 2.22$ y los $24.86 \pm 2.8$ años, respectivamente, de los cuales hemos tomado como muestra la huella plantar del pie derecho.

Los participantes del estudio acudieron al Área Clínica de Podología de la Universidad de Sevilla durante un periodo comprendido entre julio de 2014 y septiembre de 2014, donde se les informó y entregó un documento de consentimiento informado sobre los objetivos y posibles riesgos de esta investigación. Este estudio cumple todos los requisitos bioéticos de investigación con humanos según la Declaración de Helsinki, y que fue aprobado por la Dirección del Área Clínica de Podología de la Universidad de Sevilla.

Como criterio de inclusión, pacientes sanos de edades comprendidas entre los 18 y los 40 años; establecemos como límite de edad los 40 años, porque a partir de esta edad se calcula que comienza a deteriorarse la AGP ${ }^{6}$. Y los criterios de exclusión fueron pacientes con artritis reumatoide, diabetes, neuropatía o trastorno vascular, presencia de cualquier alteración dérmica que pudiera alterar la imagen de la huella, pacientes que presentasen síndrome de hiperlaxitud ligamentosa, antecedente de cirugía osteoarticular en el pie, personas con problemas de estabilidad, y que requiriesen de dispositivos ortopédicos.

Las variables que hemos considerado para tener en cuenta en el estudio son: edad, peso, sexo, altura, índice de masa corporal, FPI, ángulo de Clarke, índice de Chippaux-Smirack, índice de Staheli, anchura del antepié, anchura del itsmo, anchura del talón. El Foot Posture Index (FPI) o Índice postural del pie (IPP) es una herramienta clínica diagnóstica cuya finalidad es cuantificar el grado de posición neutra, pronada o supinada del pie. Se trata de un método simple de puntuación de varios factores de la postura del pie por medio de un resultado simple y cuantificable ${ }^{17}$. La puntuación está comprendida entre $-12 y+12$. Los valores alrededor de 0 coincidirán con un pie neutro, siendo a su vez los de un pie pronado los que se acerquen $\mathrm{a}+12$, y de un pie supinado los que lo hagan a -12 .

Para la obtención de la huella se utiliza el escáner digital plantar CbsScanFoot modelo EDP-G2-A, avalando su fiabilidad intra e interobservadora para el registro de la huella plantar el trabajo de Papuga y cols. ${ }^{18}$. 


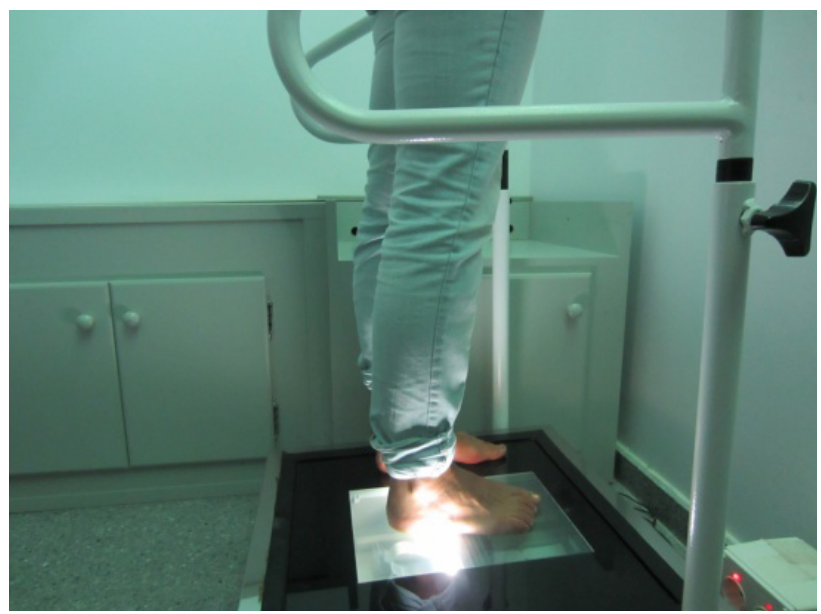

Figura 1. Obtención huella carga bipodal.

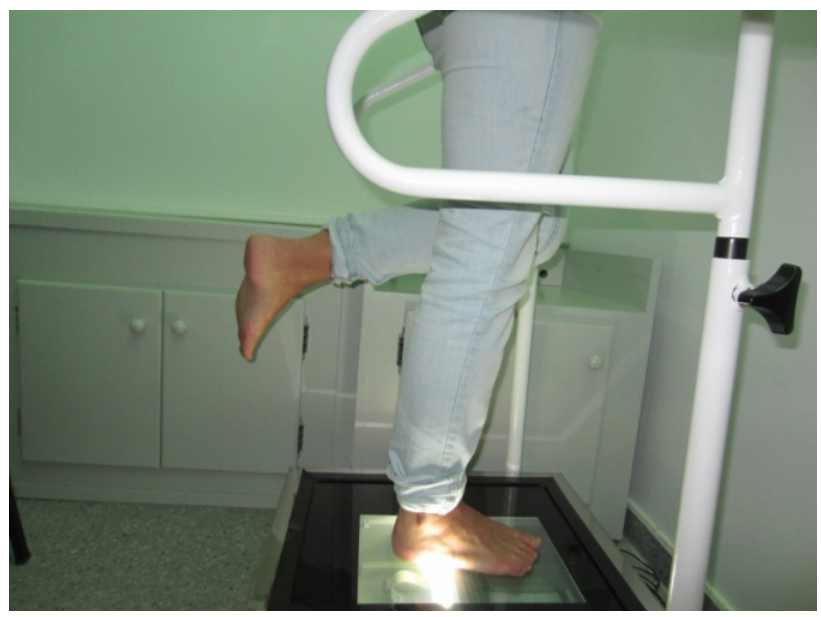

Figura 2. Obtención huella carga monopodal.

A cada paciente se le obtiene la huella en diferentes posiciones:

a) En carga bipodal: paciente en posición bípeda, con la mirada al frente y con los brazos relajados, respetando su ángulo y base de marcha, para que la obtención de la huella sea lo más real a su posición en estática (Figura 1).

b) En carga monopodal: paciente en posición bípeda, con la mirada al frente, y agarrándose para mantener su estabilidad en unas barandas laterales. Para obtener la huella se le pide al paciente que flexione la rodilla izquierda, de manera que solo contacte con el suelo el pie derecho (Figura 2).

c) En sedestación: paciente sentado en una silla con la espalda pegada al respaldo, los brazos en posición de $90^{\circ}$ aproximadamente, de manera que las manos se sitúen en el muslo, con las rodillas en flexión, en posición relajada y con el pie en ángulo recto en relación con el suelo del escáner (Figura 3).

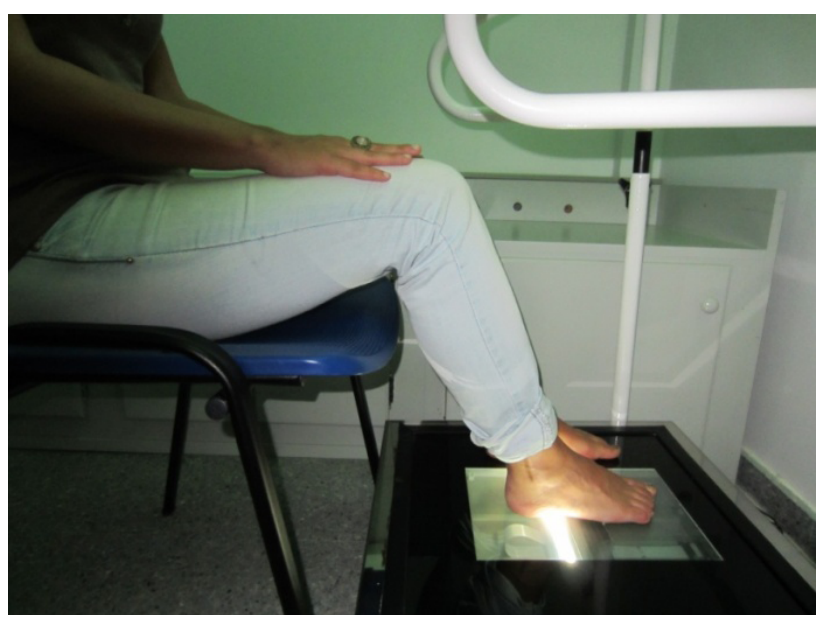

Figura 3. Obtención huella sedestación.

Una vez obtenidas las huellas plantares se procedió a la medición de las variables sobre las mismas. Para llevar a cabo dicha tarea se utilizó el programa AutoCAD ${ }^{\circledR}$, con el que procedimos a la realización de los cálculos de las diferentes medidas: ángulo de Clarke, índice de Chippaux-Smirack, índice de Staheli, anchura del antepié, anchura del itsmo y anchura del talón.

El análisis estadístico se realizó con el paquete estadístico SPSS versión 18 para Windows. Se describe la muestra total, atendiendo a edad, sexo, IMC e IPP. Se describen las mediciones tomadas en la huella tanto en bipedestación bipodal, bipedestación monopodal y sedestación. Se utilizó el test de Shapiro-Wilks para determinar la normalidad de la muestra. Para la comparación entra las distintas mediciones y su posición se ha realizado la $t$ de Student para las variables ángulo de Clarke, índice de Chippaux-Smirrack, índice de Staheli, anchura del antepié, anchura del istmo, anchura del talón. Para estudiar las posibles correlaciones entre las variables, se utilizó coeficiente de correlación de Pearson.

\section{RESULTADOS}

La muestra estudiada estaba compuesta por 14 personas, 7 hombres y 7 mujeres, siendo la edad media de la misma $24.43 \pm 2.22$ y los $24.86 \pm 2.8$ años, respectivamente. El IMC medio de las personas estudiadas era $22.50 \pm 2.79$.

Respecto al FPI la media para sujetos pronadores era $7.5 \pm$ 1.08, 1 para neutros y $-2 \pm 1$ para supinadores. La Tabla I muestra la estadística descriptiva de las variables estudiadas en cada una de las posiciones.

Posteriormente, se aplicó la t de Student para muestras relacionadas, a fin de determinar si existían diferencias significativas entre las variables estudiadas en cada una de las posiciones, así como coeficiente de Pearson para estudiar la correlación entre las mismas (Tabla II y III). 
Tabla I. Estadística descriptiva de las variables en las tres posiciones.

\begin{tabular}{lccc} 
& Bipedestación bipodal & Bipedestación monopodal & Sedestación \\
\hline & Media $\pm \mathrm{DE}$ & Media $\pm \mathrm{DE}$ & Media $\pm \mathrm{DE}$ \\
\hline Ángulo de Clarke & $56.4 \pm 6.47$ & $53.41 \pm 7.47$ & $73.35 \pm 5.97$ \\
\hline Índice de Chippaux-Smirack & $20.56 \pm 6.48$ & $24.86 \pm 7.77$ & $16.47 \pm 4.32$ \\
\hline Índice de Staheli & $0.36 \pm 0.12$ & $0.43 \pm 0.15$ & $0.31 \pm 0.08$ \\
\hline Anchura del antepié & $9.13 \pm 0.57$ & $9.23 \pm 0.64$ & $8.82 \pm 0.58$ \\
\hline Anchura del istmo & $1.89 \pm 0.67$ & $2.31 \pm 0.81$ & $1.44 \pm 0.35$ \\
\hline Anchura del talón & $5.05 \pm 0.53$ & $5.22 \pm 0.51$ & $4.55 \pm 0.41$ \\
\hline
\end{tabular}

DE: desviación estándar.

Tabla II. T-Student y coeficiente de Pearson

en las variables ángulo de Clarke, índice de

Chippaux-Smirrack e índice de Staheli

\begin{tabular}{lccccccc} 
& \multicolumn{2}{c}{$\begin{array}{c}\text { Ángulo } \\
\text { de Clarke }\end{array}$} & \multicolumn{2}{c}{$\begin{array}{c}\text { Índice de } \\
\text { Chippaux- } \\
\text { Smirrack }\end{array}$} & \multicolumn{2}{c}{$\begin{array}{c}\text { Índice } \\
\text { de Staheli }\end{array}$} \\
\hline & $\mathrm{p}$ & $\mathrm{r}$ & $\mathrm{p}$ & $\mathrm{r}$ & $\mathrm{p}$ & $\mathrm{r}$ \\
\hline $\begin{array}{l}\text { B. Bipodal- } \\
\text { B. Monopodal }\end{array}$ & 0.02 & 0.83 & $<0.01$ & 0.89 & $<0.05$ & 0.89 \\
\hline $\begin{array}{l}\text { B. Monopodal- } \\
\text { Sedestación }\end{array}$ & $<0.01$ & 0.17 & $<0.05$ & -0.13 & 0.02 & -0.10 \\
\hline $\begin{array}{l}\text { Sedestación- } \\
\text { B. Bipodal }\end{array}$ & $<0.01$ & 0.38 & 0.05 & 0.10 & 0.19 & 0.11 \\
\hline
\end{tabular}

Tabla III. T-Student y coeficiente de Pearson en las variables anchura de antepié, anchura del itsmo, anchura de talón

\begin{tabular}{lcccccccc} 
& \multicolumn{2}{c}{$\begin{array}{c}\text { Anchura } \\
\text { de antepié }\end{array}$} & \multicolumn{2}{c}{$\begin{array}{c}\text { Anchura } \\
\text { del itsmo }\end{array}$} & \multicolumn{2}{c}{$\begin{array}{c}\text { Anchura } \\
\text { de talón }\end{array}$} \\
\hline & $\mathrm{p}$ & $\mathrm{r}$ & $\mathrm{p}$ & $\mathrm{r}$ & $\mathrm{p}$ & $\mathrm{r}$ \\
\hline $\begin{array}{l}\text { B. Bipodal- } \\
\text { B. Monopodal }\end{array}$ & 0.02 & 0.98 & $<0.01$ & 0.91 & $<0.02$ & 0.95 \\
\hline $\begin{array}{l}\text { B. Monopodal- } \\
\text { Sedestación }\end{array}$ & 0.001 & 0.96 & $<0.03$ & -0.02 & $<0.01$ & 0.79 \\
\hline $\begin{array}{l}\text { Sedestación- } \\
\text { B. Bipodal }\end{array}$ & 0.001 & 0.98 & 0.03 & 0.18 & $<0.01$ & 0.89 \\
\hline
\end{tabular}

Tabla IV. Correlación FPI con ángulo de Clarke, índice de Chippaux-Smirrack e índice de Staheli en sus diferentes posiciones

\begin{tabular}{lccc} 
Correlación Pearson & Bipedestación bipodal & Bipedestación monopodal & Sedestación \\
\hline FPI y Ángulo de Clarke & 0.52 & 0.69 & 0.36 \\
\hline FPI e Índice de Chippaux-Smirrack & -0.12 & -0.11 & -0.45 \\
\hline FPI e Índice de Staheli & -0.76 & -0.12 & -0.47 \\
\hline
\end{tabular}

Para el ángulo de Clarke en las diferentes posiciones observamos diferencias significativas en todas las mediciones $(p<0.05)$. En relación con las correlaciones en la modalidad bipodal-monopodal se hallaron una relación fuerte $(r=0.83)$.

En el índice de Chippaux-Smirrack obtuvimos diferencias estadísticamente significativas en todas las mediciones $(p<0.05)$. En relación con las correlaciones en la modalidad bipodal-monopodal se hallaron una relación fuerte $(r=0.89)$.

En el índice de Staheli en las diferentes posiciones obtuvimos diferencias estadísticamente significativas $(p<0.05)$, excepto la comparación entre sedestación y bipedestación bipodal. En relación con las correlaciones en la modalidad bipodal-monopodal se hallaron una relación fuerte $(r=0.89)$.

Para las anchuras del antepié, del istmo y del talón en las diferentes posiciones, obtuvimos diferencias estadísticamen- te significativas en todas las mediciones $(p<0.05)$. Respecto al coeficiente de Pearson, los valores fueron muy altos para la anchura del antepié y de retropié, entre todas las posiciones; para la anchura del itsmo el valor fue alto al comparar bipodal y monopodal.

Al analizar la correlación entre el FPI y el ángulo de Clarke, índice de Chippaux-Smirrack y el índice de Staheli en cada una de las posiciones estudiadas, observamos que existe correlación fuerte directa entre FPI y ángulo de Clarke en carga monopodal ( $r=0.69$ ). Por otro lado, observamos correlación fuerte inversa entre FPI y ángulo de Staheli en carga bipodal (Tabla IV).

Por último, se calculó el porcentaje de variación respecto a las variables estudiadas entre la posición en carga monopodal y bipodal (Tabla V). 
Tabla V. Variación de porcentajes entre bipedestación bipodal y monopodal

\begin{tabular}{lcccc} 
& Bipedestación bipodal & Bipedestación monopodal & Diferencia & $\%$ \\
\hline Ángulo de Clarke & $56.4^{\circ}$ & $53.41^{\circ}$ & $2.99^{\circ}$ & $-5.30 \%$ \\
\hline Índice de Chippaux-Smirrack & $20.56 \%$ & $24.86 \%$ & $-4.3 \%$ & $20.91 \%$ \\
\hline Índice de Staheli & $0.36 \%$ & $0.43 \%$ & $-0.07 \%$ & $19.44 \%$ \\
\hline Anchura del antepié & $9.13 \mathrm{~cm}$ & $9.23 \mathrm{~cm}$ & $-0.10 \mathrm{~cm}$ & $1.09 \%$ \\
\hline Anchura del istmo & $1.89 \mathrm{~cm}$ & $2.31 \mathrm{~cm}$ & $-0.42 \mathrm{~cm}$ & $22.22 \%$ \\
\hline Anchura del talón & $5.05 \mathrm{~cm}$ & $5.22 \mathrm{~cm}$ & $-0.17 \mathrm{~cm}$ & $3.36 \%$ \\
\hline
\end{tabular}

\section{DISCUSIÓN}

El registro de las variaciones en la huella plantar en relación con la posición del paciente puede ser una forma de determinar si el paciente puede desarrollar patologías a nivel del pie. Las metatarsalgias y los síndromes dolorosos del talón son patologías frecuentes de consulta, cuyo origen a veces es desconocido ${ }^{5,9}$. El poder determinar si la grasa plantar ha perdido sus características amortiguadoras ayudaría a comprender el origen de estas patologías ${ }^{4,8-11}$.

Determinar la huella plantar a través de un escáner es un método rápido para informatizar la huella y utilizar medios digitales para su medición. Los estudios se centran principalmente en la observación de los cambios producidos en el grosor y sus propiedades y, de esta forma, es sencillo poder llevarlo a cabo 1,3,4,8-12.

Se observó que se producía un aumento en los valores de la anchura del antepié y del talón a medida que aumentaba la carga. En el estudio forense realizado por Reel y cols. ${ }^{19}$, cuyo objetivo era estimar la altura de individuos a partir de mediciones antropométricas del pie; se realizaron pedigrafías en estática y dinámica y se calculó la anchura del antepié y del talón, obteniéndose una media de $93.22 \pm 7.39 \mathrm{~mm}$ y 49.68 $\pm 5.33 \mathrm{~mm}$, respectivamente. En relación con los valores de nuestro estudio, observamos que aumentaban a medida que se incrementaba la carga y que, además, los obtenidos en carga monopodal se asemejaban a los obtenidos por Reel en dinámica, por lo que podemos interpretar que la expansión del pie en carga monopodal y en dinámica son similares, pudiendo estudiar cómo se comportaría en pie en dinámica observándolo en carga monopodal.

En cuanto al ángulo de Clarke, las medias obtenidas en nuestro estudio fueron $73,35 \pm 0,08^{\circ}$ en sedestación, 56,4 \pm $6,47^{\circ}$ en carga bipodal y $53,41 \pm 7,47^{\circ}$ en carga monopodal. De esta forma observamos que se producía un aplanamiento del arco a medida que aumenta la carga, lo cual corrobora nuestra hipótesis, variando así la huella en las diferentes posiciones. Según las clasificaciones de Bavor y Horawa (1974) y Jawroski (1987) de $0-29^{\circ}$ pie aplanado, $30-50^{\circ}$ se trataría un pie normal y a partir de los $50^{\circ}$ podría asociarse a un pie cavo ${ }^{20}$.

En el trabajo de Shiang y cols. ${ }^{21}$ en el que se evaluaron diferentes parámetros predictores de la altura del arco, las huellas se obtuvieron mediante plataforma de presiones y en bipedestación bipodal. En relación al ángulo de Clarke obtuvieron una media de $46,29 \pm 9,75^{\circ}$. Si los comparamos con los datos de nuestro estudio, observamos que nuestra media era superior en $10^{\circ}$ aproximadamente, lo cual se pudo deber a que la plataforma utilizada en el estudio de Shiang y cols. ${ }^{21}$ estaba compuesta por sensores que ante presiones muy bajas, como son las que se producen en las zonas marginales de la huella, no las registra dando una imagen de huella más cava que la que registra el escáner utilizado por nosotros, por lo que sus valores del ángulo de Clarke van a ser menores.

En el trabajo realizado por López y cols. ${ }^{22}$ se estudiaron las huellas de jóvenes jugadores de fútbol y no jugadores. Las huellas se obtuvieron a través de pedigrafía, y siempre en bipedestación bipodal. Respecto al ángulo de Clarke, en el pie derecho fue de $47,8 \pm 9,8^{\circ}$. Podríamos considerar las variaciones de estos datos principalmente asociados a la diferencia de utilización de pedigrafía en vez de podoscopio electrónico, ya que como recoge el trabajo de Fascione y cols. ${ }^{23}$, los datos recogidos con diversas técnicas demostraban diferencias, por lo que no se recomienda el uso intercambiable de los valores obtenidos a partir de los diferentes métodos de obtención de la huella. A pesar de ello, existen menos diferencias si comparamos nuestros datos con los de López y cols. ${ }^{22}$ que con los de Shiang y cols. ${ }^{21}$, lo cual confirma la idea de que la huella registrada por plataformas quizás no sea real.

Respecto al índice de Chippaux-Smirrack las medias obtenidas en nuestro estudio fueron en sedestación 16,47 $\pm 4,32 \%$, en carga bipodal $20,56 \pm 6,48 \%$, y en carga monopodal 24,86 $\pm 7,77 \%$. Al igual que ocurre con el ángulo de Clarke, a medida que aumenta la carga la huella se va aplanando y cambiando. En el trabajo realizado por López y cols. ${ }^{22}$ también se evaluó el índice de Chippaux-Smirrack. Las medias que se obtuvieron fuero de $31 \pm 9,5 \%$. Por su parte en el estudio realizado por Shiang y cols. ${ }^{21}$ también se evaluó el índice de Chippaux-Smirrack, obteniéndose unas medias de $68 \pm 21 \%$. Al igual que en relación con el ángulo de Clarke, nuestros datos no son comparables con los de los otros estudios, ya que se utiliza un instrumento de obtención de huella diferente.

En nuestro estudio obtuvimos valores del índice de Staheli de 0,31 $\pm 0,08 \%$ en sedestación, $0,36 \pm 0,12 \%$ en carga bipodal y $0,43 \pm 0,15 \%$ en carga monopodal. En el trabajo de 
Shiang y cols. ${ }^{21}$ también se utilizó como predictor del arco el índice de Staheli, obteniéndose una media de 0,82 \pm 0,24 \%. También se puede decir, al igual que ocurre en los casos del ángulo de Clarke o índice de Chippaux-Smirrack, que lo datos obtenidos no son comparables al utilizar métodos de obtención de huella diferentes.

Es significativo que, tanto en el estudio de Shiang como en el nuestro, los valores medios obtenidos indican huellas de pies cavos según los valores normales, por lo que nos planteamos si actualmente por diversos factores hay una tendencia a la elevación del arco de forma fisiológica.

Al aplicar la $t$ de student para muestras dependientes, encontramos en cada una de las posiciones que existía una diferencia estadística significativa $(p<0,05)$ tanto en el ángulo de Clarke como en el índice de Chippaux-Smirrack y el de Staheli, salvo en este último en la comparación entre sedestación y bipedestación bipodal $(p=0,188)$. Ello indica que la expansión que sufren las partes blandas fue significativa a la hora de la dinámica.

Al aplicar la t de Student en todas las mediciones de la anchura del antepié, istmo y talón en las diferentes posiciones se observaron diferencias significativas $(p<0,05)$, así como correlaciones fuertes en las tres medidas en sus diferentes posiciones, salvo en la anchura del istmo en las modalidades de sedestación.

Se observó una correlación fuerte directa entre las variables del FPI y ángulo de Clarke, salvo en sedestación. Como ya comentamos el ángulo de Clarke mide de forma indirecta la altura del arco longitudinal interno ${ }^{24}$, y la altura y la congruencia del arco es uno de los parámetros utilizados por el FPI. Es por ello por lo que podemos decir que el cálculo del ángulo de Clarke, a partir de la obtención de la huella en bipedestación bipodal o monopodal, estuvo correlacionado con el FPI.

Por ello, podríamos decir de entre los tres, que el que mejor nos puede predecir la altura del arco, ya que es más fiable en correlación al FPI, es el ángulo de Clarke, siempre y cuando obtengamos las huellas plantares en carga bipodal o monopodal.

A partir de los datos obtenidos, podemos calcular el porcentaje de modificación que sufren las variables a medida que aumentaba la carga. En la anchura del antepié se observó un aumento (1,09\%) debido a que al aumentar la carga la grasa plantar se expandió. En este apartado reside la importancia de la misma a la hora de padecer metatarsalgia. Una no variación de la expansión de la misma, o por el contrario una expansión exagerada, aumentaría los picos de presión a nivel metatarsal. Respecto a este tema podemos contraponer los estudio de Waldecker ${ }^{10}$ y Abouaesha y cols. ${ }^{25}$. El primero concluyó en su trabajo que no había asociación entre una disminución del espesor del AGP y las metatarsalgias, ni con la intensidad ni la frecuencia de las mismas. El segundo concluyó que existía una fuerte relación inversa entre el grosor del AGP y mediciones dinámicas de presión del pie. Nosotros nos posicionaríamos más a favor de este último porque pueden aparecer patologías en la zona metatarsal si no mantenemos en correcto estado la grasa plantar. Esto sería como consecuencia de la pérdida de la capacidad de amortiguación.

A nivel del istmo se observó un aumento $22 \%$, siendo la estructura que mayor variación sufrió debido a que, como estructura de soporte que es, se verá más solicitada a medida que aumentemos la carga ensanchándose. En el talón la anchura aumentó también (3,36 \%) debido a la importancia de la expansión de la grasa plantar. Respecto a esto, y al igual que a nivel metatarsal, el mal funcionamiento de dicha estructura a este nivel podría generar patologías ${ }^{8}$. También se podría explicar que se produce un mayor aumento de la expansión a nivel del talón $(3,36 \%$ ) que del antepié $(1,09)$, principalmente debido al grosor diferente de las estructuras a este nivel. A nivel metatarsal se calcula que es de entre 0,5 y $1,5 \mathrm{~mm}^{9,10,26}$ y a nivel del talón entre 1,5 y $1,8 \mathrm{~mm}^{27-29}$. Ello evidencia el papel amortiguador tan importante que tiene el talón en la fase de contacto de talón y la función más propulsiva que amortiguadora del antepié en la fase de despegue.

Los resultados del estudio sugieren que existen cambios en la huella plantar en las diferentes posiciones. Se observan cambios en la misma a medida que aumenta la carga fruto del comportamiento de las estructuras músculo-esqueléticas y de la AGP. Respecto a esta última, el estudio, al utilizar personas jóvenes y sanas, se ha intentado presentar el comportamiento que esta manifiesta dentro de la normalidad.

Las limitaciones de este trabajo fueron el bajo tamaño muestra y el haber contado con pacientes jóvenes sanos.

En posteriores estudios sería interesante comparar dichos comportamientos con los de grupos de población en los que la AGP pudiera estar afectada (diabéticos, neuropatía, trastornos vasculares) o con grupos de población de edad avanzada, en la que la AGP comienza a degenerarse. Dicha comparación también podría acercar a la realidad sobre si los cambios que se producen en la AGP se correlacionan con diversas patologías (metatarsalgia, ulceración, talalgia).

Otro aspecto que resulta interesante y que se incluirá en futuros estudios es comparar el comportamiento que sufre el pie a nivel prono-supinatorio, pudiéndose utilizar parámetros como la línea de Helbing, que nos permitiera observar los grados de pronación del retropié, y si estos influyen en mayor o menor medida en las modificaciones que sufre la huella plantar.

Como conclusiones, hemos determinado que la grasa plantar a nivel del talón sufre una expansión $0,17 \mathrm{~cm}$, el antepié $0,10 \mathrm{~cm}$ y el itsmo $0,42 \mathrm{~cm}$; asimismo el ángulo de Clarke disminuye $2,99^{\circ}$, el índice de Chippaux-Smirrack aumenta 4,3\% y el índice de Staheli aumenta 0,07\%, todos ellos al pasar de un estado de carga bipodal a monopodal. Al comparar el FPI con las variaciones observadas, destacamos la correlación entre dicho parámetro con el ángulo de Clarke en carga monopodal. Ello se entiende dado que el 71,4\% de los sujetos eran pronadores y, al estar en máxima carga (monopodal), el ángulo reflejó esa pronación. Lo mismo no ocurre con los índices de Staheli ni de Chippaux-Smirrack, ya 
que estas variables se obtienen de la relación entre el itsmo y el talón o el antepié respectivamente y dichas anchuras no tienen por qué modificarse al pronar.

\section{CONFLICTO DE INTERESES}

Los autores no presentan ningún tipo de conflicto de interés.

\section{FUENTES DE FINANCIACIÓN}

Este trabajo de investigación no ha recibido financiación por parte de ninguna entidad.

\section{BIBLIOGRAFÍA}

1. Aerts P, Ker RF, De Clercq D, Ilsley DW, Alexander RM. The mechanical properties of the human heel pad: a paradox resolved. J Biomech. 1995;28(11):1299-308. DOI: 10.1016/0021-9290(95)00009-7.

2. Perry J. Anatomy and biomechanics of the hindfoot. Clin Orthop Relat Res. 1983;(177):9-15.

3. Ozdemir H, Söyüncü $Y$, Ozgörgen M DK. Efectos de los cambios en el grosor y elasticidad de la almohadilla grasa del talón en el dolor de talón. Podol clínica. 2005;6(4):114-9.

4. Prichasuk S. The heel pad in plantar heel pain. J Bone Joint Surg Br. 1994;76(1):140-2.

5. Coughlin MJ, Mann RA SC. Pie y tobillo. Madrid: Marbán, editor; 2001.

6. Jahss MH, Michelson JD, Desai P, Kaye R, Kummer F, Buschman W, et al. Investigations into the fat pads of the sole of the foot: anatomy and histology. Foot Ankle. 1992;13(5):233-42.

7. Quirk R. Metatarsalgia. Aust Fam Physician. 1996;25(6):863-9.

8. Tsai WC, Wang CL, Hsu TC, Hsieh FJ, Tang FT. The mechanical properties of the heel pad in unilateral plantar heel pain syndrome. Foot ankle Int. 1999;20(10):663-8. DOI: 10.1177/107110079902001010.

9. Waldecker U. Plantar fat pad atrophy: a cause of metatarsalgia? J Foot Ankle Surg. 2001;40(1):21-7. DOI: 10.1016/s1067-2516(01)80037-5.

10. Waldecker $U$, Lehr HA. Is there histomorphological evidence of plantar metatarsal fat pad atrophy in patients with diabetes? J Foot Ankle Surg. 2009;48(6):648-52. DOI: 10.1053/j.jfas.2009.07.008.

11. Prichasuk $S$, Mulpruek $P$, Siriwongpairat $P$. The heel-pad compressibility. Clin Orthop Relat Res. 1994;(300):197-200.

12. Gooding GA, Stess RM, Graf PM, Moss KM, Louie KS, Grunfeld C. Sonography of the sole of the foot. Evidence for loss of foot pad thickness in diabetes and its relationship to ulceration of the foot. Invest Radiol. 1986;21(1):45-8.

13. Hill CL, Gill TK, Menz HB, Taylor AW. Prevalence and correlates of foot pain in a population-based study: the North West Adelaide health study. J Foot Ankle Res. 2008; 1(1):2. DOI: 10.1186/1757-1146-1-2.
14. Dunn JE, Link CL, Felson DT, Crincoli MG, Keysor JJ, McKinlay JB. Prevalence of foot and ankle conditions in a multiethnic community sample of older adults. Am J Epidemiol. 2004;159(5):491-8. DOI: 10.1093/aje/ kwh071.

15. Hsu C-C, Tsai W-C, Hsiao T-Y, Tseng F-Y, Shau Y-W, Wang C-L, et al. Diabetic effects on microchambers and macrochambers tissue properties in human heel pads. Clin Biomech (Bristol, Avon). 2009;24(8):682-6. DOI: 10.1016/j.clinbiomech.2009.06.005.

16. Argimón JM, Chinchilla $\mathrm{A}$, Forbes $\mathrm{CH}$, Kiley R. Métodos de investigación clínica y epidemiológica. JANO, Med y Humanidades. 33:30.

17. Redmond AC, Crosbie J, Ouvrier RA. Development and validation of a novel rating system for scoring standing foot posture: The Foot Posture Index. Clin Biomech. 2006;21(1):89-98. DOI: 10.1016/j.clinbiomech.2005.08.002.

18. Papuga MO, Burke JR. The reliability of the Associate Platinum digital foot scanner in measuring previously developed footprint characteristics: a technical note. J Manipulative Physiol Ther. 2011;34(2):114-8. DOI: 10.1016/j.jmpt.2010.12.008.

19. Reel S, Rouse S, Vernon W, Doherty P. Estimation of stature from static and dynamic footprints. Forensic Sci Int. 2012;219(1-3):283.e1-283. e5. DOI: 10.1016/j.forsciint.2011.11.018.

20. Buenhombre M. Estudio de la huella plantar estática en niños practicantes de fútbol. Trabajo de Grado. Universidad de Salamanca; 2000.

21. Shiang TY, Lee SH, Lee SJ, Chu WC. Evaluating different footprint parameters as a predictor of arch height. IEEE Eng Med Biol Mag. 1998;17(6):62-6. DOI: 10.1109/51.731323.

22. López N, Alburquerque F, Santos M, Sánchez M DR. Evaluation and analysis of the footprint of young individuals. A comparative study between football players and non-players. Eur J Anat. 2005;9(3):135-42.

23. Fascione JM, Crews RT, Wrobel JS. Dynamic footprint measurement collection technique and intrarater reliability: ink mat, paper pedography, and electronic pedography. J Am Podiatr Med Assoc. 2012;102(2):1308. DOI: $10.7547 / 1020130$.

24. Wozniacka R, Bac A, Matusik S, Szczygiel E, Ciszek E. Body weight and the medial longitudinal foot arch: high-arched foot, a hidden problem? Eur J Pediatr. 2013;172(5):683-91. DOI: 10.1007/s00431-013-1943-5.

25. Abouaesha F, van Schie CH, Griffths GD, Young RJ, Boulton AJ. Plantar tissue thickness is related to peak plantar pressure in the highrisk diabetic foot. Diabetes Care. 2001;24(7):1270-4. DOI: 10.2337/ diacare.24.7.1270.

26. Mickle KJ, Munro BJ, Lord SR, Menz HB, Steele JR. Soft tissue thickness under the metatarsal heads is reduced in older people with toe deformities. J Orthop Res. 2011;29(7):1042-6. DOI: 10.1002/jor.21328.

27. Uzel M, Cetinus E, Ekerbicer HC, Karaoguz A. Heel pad thickness and athletic activity in healthy young adults: a sonographic study. J Clin Ultrasound. 2006;34(5):231-6. DOI: 10.1002/jcu.20230.

28. Hsu CC, Tsai WC, Wang CL, Pao SH, Shau YW, Chuan YS. Microchambers and macrochambers in heel pads: are they functionally different? J Appl Physiol (Bethesda, Md 1985). 2007;102(6):2227-31. DOI: 10.1152/japplphysiol.01137.2006.

29. Zheng YP, Choi YK, Wong K, Chan S, MakAF. Biomechanical assessment of plantar foot tissue in diabetic patients using an ultrasound indentation system. Ultrasound Med Biol. 2000;26(3):451-6. DOI: 10.1016/ s0301-5629(99)00163-5. 